\title{
Emotion-Network Density in Major Depressive Disorder
}

Clinical Psychological Science 2015, Vol. 3(2) 292-300 (C) The Author(s) 2014 Reprints and permissions: sagepub.com/journalsPermissions.nav DOI: $10.1177 / 2167702614540645$ cpx.sagepub.com (SAGE

\author{
Madeline Lee Pe ${ }^{1}$, Katharina Kircanski ${ }^{2}$, Renee J. Thompson ${ }^{3}$, \\ Laura F. Bringmann', Francis Tuerlinckx', Merijn Mestdagh', \\ Jutta Mata ${ }^{4}$, Susanne M. Jaeggi ${ }^{5}$, Martin Buschkueh1 ${ }^{6}$, \\ John Jonides ${ }^{7}$, Peter Kuppens ${ }^{1}$, and Ian H. Gotlib ${ }^{2}$ \\ ${ }^{1}$ Department of Psychology, KU Leuven; ${ }^{2}$ Department of Psychology, Stanford University; ${ }^{3}$ Department \\ of Psychology, Washington University in St. Louis; ${ }^{4}$ Max Planck Institute for Human Development; \\ ${ }^{5}$ School of Education, University of California, Irvine; ${ }^{6}$ MIND Research Institute, Irvine, California; and \\ ${ }^{7}$ Department of Psychology, University of Michigan, Ann Arbor
}

\begin{abstract}
Major depressive disorder (MDD) is a prevalent disorder involving disturbances in mood. There is still much to understand regarding precisely how emotions are disrupted in individuals with MDD. In this study, we used a network approach to examine the emotional disturbances underlying MDD. We hypothesized that compared with healthy control individuals, individuals diagnosed with MDD would be characterized by a denser emotion network, thereby indicating that their emotion system is more resistant to change. Indeed, results from a 7-day experience sampling study revealed that individuals with MDD had a denser overall emotion network than did healthy control individuals. Moreover, this difference was driven primarily by a denser negative, but not positive, network in MDD participants. These findings suggest that the disruption in emotions that characterizes depressed individuals stems from a negative emotion system that is resistant to change.
\end{abstract}

\section{Keywords}

depression, emotion, affective disorders

Received 10/21/13; Revision accepted 5/19/14

The adaptive nature of emotions lies in their capacity to mobilize individuals in response to changing demands and threats in the environment (Izard, 2009). In this context, investigators have hypothesized that healthy psychological functioning is characterized by emotions that change flexibly across time (Kashdan \& Rottenberg, 2010). Given this formulation, we propose that people who are diagnosed with major depressive disorder (MDD), a prevalent condition that involves primary disturbances in mood (American Psychiatric Association, 2013), are characterized by emotions that are resistant to change.

One way to examine this proposition is by using a network approach to investigate the dynamics of emotions in MDD (Cramer, Waldorp, van der Maas, \& Borsboom, 2010). This approach has recently garnered attention in attempts to elucidate the mechanisms underlying various psychiatric disorders. From a network approach, a psychiatric disorder is conceptualized as being characterized by a constellation of symptoms, in contrast to a single underlying "essence" or construct from which all other symptoms arise (Borsboom, 2008; Kendler, Zachar, \& Craver, 2011). Thus, psychiatric disorders like MDD are not necessarily best characterized by defining specific single traits (e.g., depressed mood) but, instead, by elucidating the patterns of interactions among several core symptoms (e.g., the nature of the association between depressed mood and suicidal thoughts).

We can use a network approach to examine the temporal dynamics of the emotion system that underlies MDD. In this approach, emotional changes and fluctuations in people's current emotional experiences are predicted by

\footnotetext{
Corresponding Author:

Madeline Lee Pe, Department of Psychology, KU Leuven, Tiensestraat 102, Box 3713, Leuven 3000, Belgium

E-mail: madeline.pe@ppw.kuleuven.be
} 
different emotions experienced at a previous time point and possible external influences. The patterns of emotions that are mutually predictive can differ across individuals (Pe \& Kuppens, 2012). That is, some individuals may have stronger temporal connections among specific emotions (i.e., emotions that strongly predict each other over time) than do other individuals. The overall strength of these temporal connections represents the density ${ }^{1}$ of the emotion network (Newman, 2010): Stronger temporal connections reflect a denser emotion network, whereas weaker temporal connections reflect a less dense emotion network. For example, if we examine someone's current happiness, the extent to which this emotion is predicted by his or her anger, sadness, and happiness at the previous time point would reflect a part of the density of his or her emotion network.

This differential density can be conceptualized as an overall indicator of the resistance of the emotion system to change. A denser network suggests that emotions are more self-predictive (i.e., current emotions are predicted by emotions at a previous time point) and, thus, the less likely it is that other factors can access and influence the emotion system. This builds a constricted and insulated emotional landscape, which makes the emotion system more resistant to both internal (e.g., emotion-regulation efforts) and external (e.g., environmental) demands (Kuppens, Allen, \& Sheeber, 2010). As a result, emotions lose their adaptive function of mobilizing individuals so that they can respond to changing internal and external demands.

The proposition that depressed individuals are characterized by an emotion network that is denser and, thus, less flexible and more resistant to change than are nondepressed persons is not unique to the current study. Support for this formulation comes from studies on emotional reactivity (Rottenberg, 2005; Rottenberg, Gross, \& Gotlib, 2005) and emotional inertia (Kuppens et al., 2010) in clinically depressed individuals. In studies of emotional reactivity, it is assumed that participants' emotional responses are resistant to change when their emotional experiences do not change in response to a negative or positive external event. Indeed, it has been shown in laboratory studies that people diagnosed with MDD are characterized by emotion-context insensitivity (Rottenberg, 2005; Rottenberg et al., 2005); that is, their responses to emotional (both positive and negative) stimuli tend to be similar to their responses to neutral stimuli (for a meta-analysis, see Bylsma, Morris, \& Rottenberg, 2008). ${ }^{2}$ In turn, emotional inertia is defined as the extent to which specific emotions (or aggregated emotions, such as negative affect) persist over time and is typically measured as the autocorrelation of a single emotion over time (Kuppens et al., 2010). Higher autocorrelation reflects higher resistance to emotional change. Emotional inertia of negative emotions has been shown to be related to the severity of depressive symptoms in normal populations (Koval, Kuppens, Allen, \& Sheeber, 2012; Kuppens et al., 2012) and to the diagnosis of depression (Kuppens et al., 2010; Kuppens et al., 2012; but for an exception, see Thompson et al., 2012; see Houben, Van den Noortgate, \& Kuppens, 2013, for a meta-analysis that confirms these findings).

Although emotional inertia and density of the emotion network both reflect some form of resistance to change, they make different assumptions about what constitutes such resistance. Whereas emotional inertia indicates that the current level of an emotion is predicted by the level of that same emotion at a previous time point (whether for a single emotion, such as anger, or for an aggregated measure of emotions, such as overall negative affect), the emotion-network approach involves all mutual interrelations among different emotions by focusing on how each emotion (i.e., various positive and negative emotions) experienced at a specific time point (e.g., sadness at time $t$ ) can be predicted by all emotions at a previous time point (e.g., anger, sadness, happiness, guilt, and others at time $t-1)$. In other words, whereas inertia examines the predictability of a single emotion or affective dimension, density captures the predictability of the entire emotional system, including all interactions among different emotions. Therefore, emotional density is a much broader notion than is emotional inertia, given that it applies to the flexibility or rigidity of the dynamic interplay between the different emotional states in the system, including how they mutually influence each other across time, rather than considering single emotional states on their own.

In sum, we hypothesize that if the emotion system in MDD is resistant to change, MDD participants (relative to healthy control individuals) should have a relatively dense emotion network, which results from stronger temporal connections between different emotions. We test this hypothesis by analyzing emotion data of MDD and healthy control individuals that were obtained in a large experience sampling study.

\section{Method}

\section{Participants and procedure}

Participants were recruited from the surrounding communities of Stanford, California, and Ann Arbor, Michigan, through advertisements posted online (e.g., Craigslist) and at local businesses (e.g., bulletin boards). Participants were English-speaking adults aged 18 to 40 years. The healthy control (CTL) group ( $n=53)$ comprised individuals who experienced no current or past mental-health disorders and had scores of 9 or less on the Beck Depression Inventory-II (BDI-II; Beck, Steer, \& Brown, 
1996). The depressed (MDD) group ( $n=53)$ comprised participants who were currently diagnosed with MDD as assessed by the Structured Clinical Interview for DSM$I V-T R$ Axis I Disorders (First, Spitzer, Gibbon, \& Williams, 2001). In addition, participants in the MDD group were required to have a BDI-II score of 14 or more, an absence of alcohol-drug dependence in the past 6 months, and no Bipolar I or II diagnoses or evidence of psychotic disorders. Twenty-two individuals were excluded from analysis because of BDI-II scores being outside of the range of eligibility $(n=7)$, equipment failure $(n=12)$, or noncompliance (e.g., responding to less than 41 prompts; $n=3$ ), thereby leaving the final sample of 106 participants. The study was approved by the institutional review boards of Stanford University and University of Michigan.

Participants carried a personal digital assistant as they went about their daily activities. Palm units (Palm Pilot Z22) were individually programmed (using ESP 4.0; Barrett \& Feldman Barrett, 2000) to beep eight times a day between 10 a.m. and 10 p.m. for approximately 7 consecutive days. Prompts occurred at random times within eight 90-min windows per day; thus, prompts could occur between 2 and almost 180 min apart $(M=93$ $\min , S D=38 \mathrm{~min})$. Participants were given $3 \mathrm{~min}$ to respond to the initial question. Participants provided informed consent and were compensated for their participation, with an extra incentive for responding to more than $90 \%$ of the prompts.

Given our research question, we focus here only on the emotion items. At each beep, participants used a 4-point scale ranging from 1 (not at all) to 4 (a great deal) to indicate their current levels of negative (sad, anxious, angry, frustrated, ashamed, disgusted, guilty) and positive (happy, excited, alert, active) affect.

\section{Data preparation}

To estimate the density of the emotion network, we estimated the average temporal connection strengths among all of the emotion variables (Bringmann et al., 2013). We first conducted a series of univariate multilevel analyses with each emotion at time $t$ being predicted by all the other emotions (including the emotion variable itself) at time $t-1$, in which time $t-1$ and time $t$ refer to two consecutive beeps within the same day. This allowed us to evaluate the unique contribution of each emotion at time $t-1$ on the specific emotion of interest at time $t$. All predictors were centered on each individual's mean score. For example, the Level 1 regression equation for person $i$ for current sadness experience is as follows:

$$
\begin{aligned}
\operatorname{sad}_{i t}= & \delta_{0 i}+\delta_{1 i}\left(\operatorname{sad}_{i, t-1}\right)+\delta_{2 i}\left(\operatorname{anxious}_{i, t-1}\right) \\
& +\delta_{3 i}\left(\operatorname{angry}_{i, t-1}\right)+\ldots \delta_{11 i}\left(\operatorname{active}_{i, t-1}\right)+e_{i t} .
\end{aligned}
$$

Each slope represents the strength of the temporal connection between emotion pairs (e.g., $\delta_{1 i}$ represents the expected change in sadness at the current time point given a 1-unit increase in sadness at the previous time point, keeping everything else constant, which is similar to the measure of sadness inertia, but controlling for the effects of the other emotions as well; $\delta_{2 i}$ represents the expected change in current sadness given a 1-unit increase in previous anxiety, while controlling for the effects of the other emotions). This procedure was conducted 11 times, once for every emotion as a dependent variable. At Level 2 of each of the multilevel models, we estimated an empty model; thus, all random effects (intercepts and slopes) are assumed to come from a multivariate normal distribution (one for each separate regression).

In the next step, individual participants' slopes were then extracted from the multilevel model. Because we were interested in the strength of these connections regardless of directionality, we calculated the average of the absolute value of the 121 slopes to represent the density of the emotion network for each person. Two participants from the CTL group were removed from analysis because their overall emotion-network values were more than 3 SDs above the mean of their group. ${ }^{3}$

We also wanted to elucidate the precise location of the emotion-network density in MDD by examining the density of the network separately for positive and negative emotions. The density of the positive emotion network is the mean of the 44 absolute slope values from the series of multilevel models that used positive emotions as the dependent variable: A positive emotion network refers to how much previous emotions predict current individual positive emotions. Similarly, the density of the negative emotion network is the mean of the 77 absolute slope values from the series of multilevel models that used negative emotions as the dependent variable: A negative emotion network refers to how much previous emotions predict current individual negative emotions.

\section{Results}

\section{Participant characteristics}

The MDD and CTL groups did not differ significantly in gender, $\chi^{2}(1, N=106)=0.18, p=.83, \phi_{\mathrm{c}}=.04(\mathrm{MDD}$ : $71.7 \%$ female, $28.3 \%$ male; CTL: $67.9 \%$ female, $32.1 \%$ male), education, $\chi^{2}(3, N=106)=6.67, p=.08, \phi_{c}=.25$ (MDD: $11 \%$ high school graduate, $38 \%$ some college, 43\% bachelor's degree, 8\% advanced degree; CTL: $47 \%$ some college, 43\% bachelor's degree, 9\% advanced degree), or ethnicity, $\chi^{2}(5, N=106)=7.79, p=.17, \phi_{\mathrm{c}}=$ .27 , with both groups being largely Caucasian (MDD: 73.6\%; CTL: 62.3\%). The two groups did differ in age, $t(104)=2.19, p=.03$, Cohen's $d=0.44$; the MDD group 
Table 1. Emotion-Network Density by Depression Status

\begin{tabular}{|c|c|c|c|c|c|}
\hline \multirow[b]{2}{*}{ Emotion network } & \multicolumn{2}{|c|}{ Depression status group } & \multicolumn{2}{|c|}{ Difference test } & \multirow{2}{*}{$\begin{array}{l}\text { Effect size } \\
\text { (Cohen's } d \text { ) }\end{array}$} \\
\hline & CTL $(n=51)$ & $\operatorname{MDD}(n=53)$ & $t(102)$ & $p$ & \\
\hline Overall & $0.05(0.00)$ & $0.08(0.00)$ & 10.08 & $<.001$ & 2.00 \\
\hline Negative & $0.04(0.00)$ & $0.09(0.00)$ & 12.34 & $<.001$ & 2.44 \\
\hline Positive & $0.07(0.00)$ & $0.07(0.00)$ & -0.26 & .79 & -0.05 \\
\hline Anxious & $0.06(0.00)$ & $0.10(0.01)$ & 5.49 & $<.001$ & 1.09 \\
\hline Sad & $0.03(0.00)$ & $0.10(0.01)$ & 10.92 & $<.001$ & 2.16 \\
\hline Disgusted & $0.04(0.00)$ & $0.10(0.01)$ & 10.44 & $<.001$ & 2.07 \\
\hline Angry & $0.04(0.00)$ & $0.08(0.01)$ & 6.63 & $<.001$ & 1.31 \\
\hline Guilty & $0.02(0.00)$ & $0.08(0.01)$ & 9.64 & $<.001$ & 1.91 \\
\hline Ashamed & $0.04(0.00)$ & $0.09(0.00)$ & 9.23 & $<.001$ & 1.83 \\
\hline Frustrated & $0.05(0.00)$ & $0.08(0.00)$ & 6.07 & $<.001$ & 1.20 \\
\hline Active & $0.06(0.00)$ & $0.06(0.00)$ & 0.63 & .53 & 0.12 \\
\hline Alert & $0.07(0.00)$ & $0.08(0.00)$ & 1.23 & .22 & 0.24 \\
\hline Happy & $0.07(0.00)$ & $0.06(0.00)$ & -1.77 & .08 & -0.35 \\
\hline Excited & $0.07(0.00)$ & $0.06(0.00)$ & -1.04 & .30 & -0.21 \\
\hline
\end{tabular}

Note: The table presents means for each measure unless otherwise indicated. Standard errors are shown in parentheses. Values in bold represent the main variables examined; these main emotion-network variables are illustrated in Figure 1. $\mathrm{CTL}=$ healthy control; MDD $=$ major depressive disorder.

$(M=28.2$ years, $S D=6.4)$ was older than the CTL group $(M=25.4$ years, $S D=6.4)$.

\section{Network density}

We conducted an independent samples $t$ test to compare the MDD and CTL groups with respect to the density of their emotion networks. We first examined their overall emotional networks, not differentiating by valence. Consistent with our hypothesis, results showed that individuals with MDD had a significantly denser overall emotion network than did the CTL individuals (see Table 1), and this difference was marked by a considerable effect size $(d=2.00)$. Because the density values in Table 1 are not readily interpretable, we illustrate this finding in Figure 1 by graphically displaying the average overall emotion network for MDD and CTL groups.

We further examined whether the two groups of participants differed in both their positive and their negative emotion networks by conducting a repeated measures analysis of variance with group (MDD, CTL) as the between-subjects variable and emotion-network valence (negative, positive) as the within-subjects factor. This analysis yielded a significant main effect of group, $F(1,102)=$ $69.15, p<.001, \eta_{p}{ }^{2}=.40$, but not of valence, $F(1,102)=$ $0.01, p=.92, \eta_{p}^{2}=.00$. These effects were qualified, however, by a significant interaction of group and valence, $F(1$, 102) $=120.58, p<.001, \eta_{p}^{2}=.54$. Follow-up $t$ tests indicated that MDD individuals had a significantly denser negative emotion network than did CTL individuals $(d=2.44)$; in contrast, MDD and CTL groups did not differ in the density of their positive emotion network ( $d=-0.05$; see Table 1 and Fig. 1)., ${ }^{4,5}$ Although not part of the main analysis, we included the density results for each emotion in Table 1 to show that the MDD and CTL groups differed significantly across all negative emotions and no positive emotions.

\section{Follow-up analyses}

Gender differences. We examined the role of gender ${ }^{6}$ by conducting a repeated measures analysis of variance similar to the one described earlier, but this time, we included gender and its interaction with group. Although there was not a main effect for gender, $F(1,100)=3.41$, $p=.07, \eta_{p}{ }^{2}=.03$, or a significant interaction of gender and group, $F(1,100)=0.40, p=.53, \eta_{p}^{2}=.00$, there was a significant interaction of valence, gender, and group, $F(1,100)=5.79, p=.02, \eta_{p}^{2}=.06$. In the MDD group, women $(M=0.10, S E=0.00)$ had a denser negative emotion network than did men $(M=0.08, S E=0.01), t(51)=$ $-2.24, p=.03, d=-0.44$; this gender difference was not evident in the CTL group, $t(49)=-0.55, p=.59, d=$ -0.11 . With respect to the density of the positive network, men and women did not differ from each other in either the MDD group, $t(51)=0.32, p=.75, d=0.06$, or the CTL group, $t(49)=-1.19, p=.24, d=-0.24{ }^{7}$

Variability of emotions. It is possible that the observed differences in the density of the emotion network can be explained by group differences in emotion variance. Indeed, the MDD individuals had a higher standard 


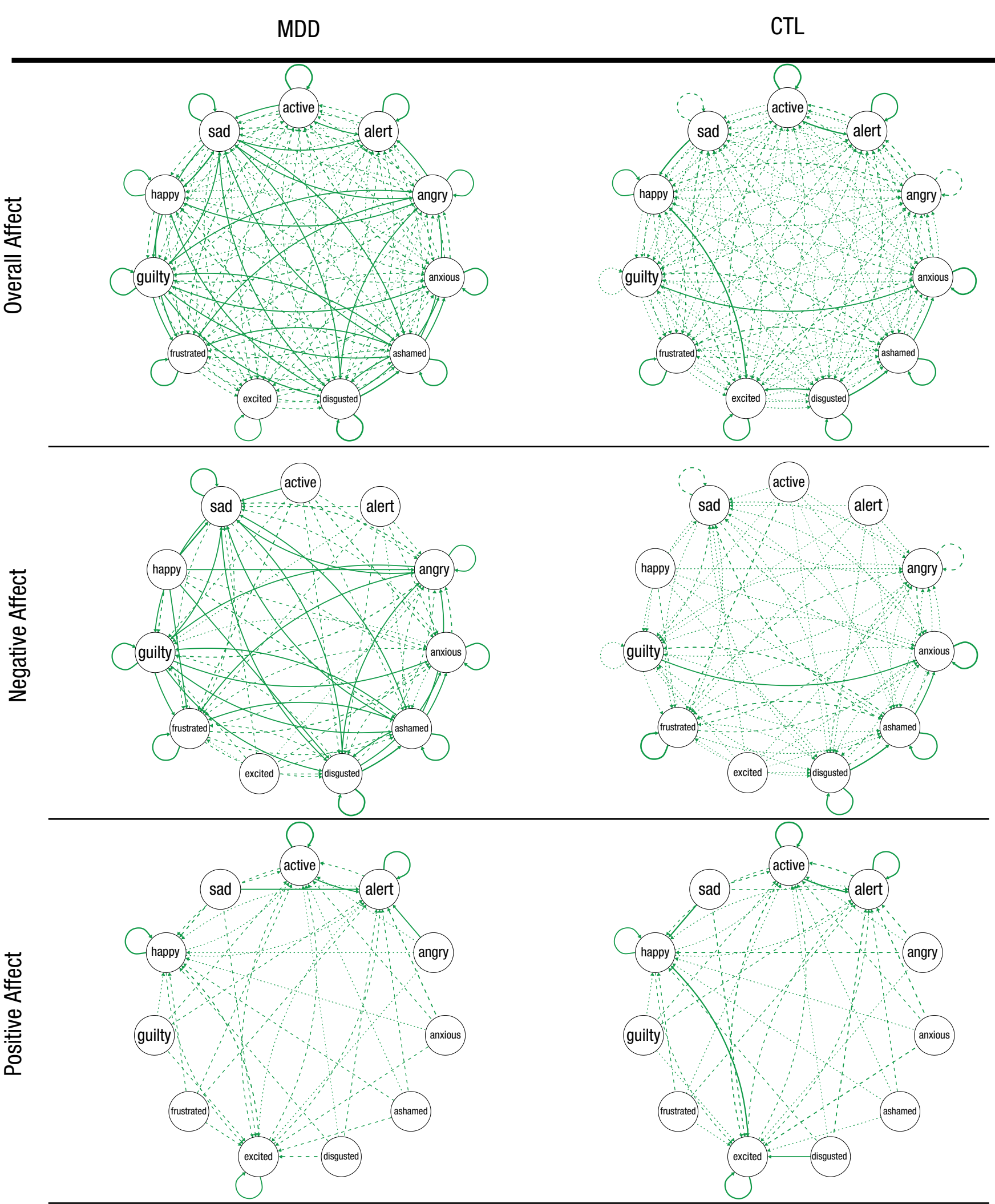

Fig. 1. Graphic display of emotion networks by depression status group. Arrows represent the strength of the connections between any two pairs of emotions (one emotion at time $t-1$ and the other at time $t$ within days). Dotted arrows correspond to slope values $1 S D$ below the mean of the overall network density across groups (weak connections), dashed arrows correspond to values around the mean across groups (values between 1 $S D$ below and above the mean of the overall network density; moderate connections), and bold arrows correspond to values $1 S D$ above the mean across groups (strong connections). MDD = depressed group; CTL $=$ healthy control group. 
deviation $^{8}$ in the intensity of their overall emotions ( $M=$ $0.69, S E=0.02)$ than did the CTL individuals $(M=0.40$, $S E=0.02), t(102)=9.83, p<.01, d=1.95$. The MDD individuals also had a higher standard deviation in their negative emotions $(M=0.72, S E=0.03)$ than did the CTL individuals $(M=0.23, S E=0.02), t(102)=13.53, p<.01$, $d=2.67$, but not in their positive emotions, $t(102)=-0.69$, $p=.49, d=-0.14$. To rule out the possibility that disproportionate variance is driving the group differences in density, we conducted a multiple regression with density as the dependent variable, and the standard deviation (standardized) and diagnostic group (MDD, CTL) as predictors. For the overall network, after we controlled for the standard deviation of overall emotions, diagnostic group remained a significant predictor of density, $\beta=$ $0.21, t(101)=3.06, p<.01, d=0.61$. Similar results were found for the negative emotion network: After we controlled for the standard deviation of negative emotions, diagnostic group remained a significant predictor of density, $\beta=0.19, t(101)=2.46, p=.02, d=0.49$. Therefore, we can conclude that the denser overall and negative emotion networks found in the MDD individuals relative to the CTL individuals were not a function of group differences in variability. For the positive emotion network, diagnostic group did not significantly predict density, $\beta=$ $0.02, t(101)=0.31, p=.76, d=0.06$, even after we controlled for the standard deviation of positive emotions. ${ }^{9}$

\section{Discussion}

Although there is a growing consensus that MDD involves disturbances of mood, it is still unclear precisely how emotions are disrupted in individuals diagnosed with MDD. In the present study, we used a network approach to examine the emotion system underlying MDD. On the basis of previous work (Kuppens et al., 2010; Rottenberg et al., 2005), we proposed that the everyday emotions of depressed individuals would be more resistant to changing internal and external demands than would the emotions of their nondepressed peers, thereby resulting in an emotion network that is more self-determining or dense. Supporting our hypothesis, our findings revealed that participants with MDD had a denser overall emotion network than did CTL individuals, which involved multiple stronger temporal connections among various emotions. Thus, compared with nondepressed control individuals, the emotions experienced by individuals with MDD tend to be more strongly determined by their previous emotions and, therefore, more resistant to change.

It is important to note that the density of this overall emotion network may be driven by the network of a particular emotion valence rather than representing a joint contribution of both positive and negative emotion networks. To investigate this possibility, we examined the network data separately for positive and negative emotions. The results of these analyses clearly indicated that the MDD and CTL groups differed in the density of their negative, but not their positive, emotion networks. ${ }^{10}$ Specifically, the MDD group had a denser negative emotion network: Their previous emotions strongly predicted their current experience of negative emotions.

Our result is consistent with previous research on emotional inertia that has indicated that it is primarily negative emotions in depression that tend to be resistant to change (Houben et al., 2013). However, it is notable that the present sample of depressed and nondepressed participants was shown to be comparable with respect to inertia of negative or positive emotions in an earlier study (Thompson et al., 2012). We believe that in reconciling this difference, it is instructive to consider that in emotional inertia, resistance to change involves the selfpredictability of a particular emotion (or an aggregated measure of emotions). In contrast, in density, resistance to change is not only a result of the self-predictability of a particular emotion (as in inertia) but also heavily influenced by the various emotions (including both positive and negative emotions) experienced by the individual. The difference in the results for these two measures suggests that the group difference in density is influenced more by the cross-lagged relations among various emotion measures than by the inertia variable. ${ }^{11}$

In addition, the emotion network quantifies resistance to change as the absolute slope values of the temporal relations between the various emotions, whereas this is not the case for emotional inertia. Thus, the directionality of the associations among emotions over time is less important when defining the density of an emotion network. A person who feels sadder after feeling angry and a person who feels less sad after feeling angry may differ qualitatively in the direction of the temporal relation between their emotions, but they may be similar in the degree of predictability of their emotions; that is, for both individuals, their previous emotions predict the intensity of their current emotions.

It is important to note here that although denser negative emotion networks did not differ by gender in the CTL group, depressed women exhibited a denser negative emotion network than did depressed men. These findings may help us to understand why women are more prone to experience depression than are men (Nolen-Hoeksema, 2001). Because depressed women's negative emotion system tends to be more resistant to change, it may be more difficult for them to break the cycle of negative emotionality. This may also help explain the heightened experience of negative emotions in women compared with men (Larsen \& Diener, 1987). These findings may also help us understand why women ruminate more than men do (Nolen-Hoeksema, Wisco, \& Lyubomirsky, 2008). Because 
rumination involves perseverative thinking about feelings, it may be that rumination arises from, contributes to, or has bidirectional influences with the more self-determining nature of negative emotions in depressed women. Future studies are needed to examine these formulations more explicitly and systematically.

Although the present findings increase knowledge of the everyday emotional functioning of individuals with MDD, this study is not without limitations. First, the MDD group was older than the CTL group; however, age did not moderate the group differences in the density of the network. Second, the positive emotion words used in this study captured only medium- or high-arousal positive emotions. To be able to draw stronger conclusions regarding group differences in positive emotion network, researchers in future studies should use a broader range of positive emotion words that are sampled across the full arousal dimension of positive emotions (Russell, 2003). Third, because the findings of the present study are based on participants who were currently diagnosed with MDD, we cannot make strong conclusions regarding their generalizability to people at risk for, or who have recovered from, depression. We suspect, however, that greater density is a trait-based risk factor for depression that would be observed in these groups as well. Future research is needed to examine this formulation.

Another possible avenue for future research is to examine the emotion network in other forms of psychopathology, such as bipolar disorder. Investigators have posited that patients with bipolar disorder experience heightened positive emotion across contexts; that is, they experience elevated levels of positive emotions not solely in response to positive or rewarding stimuli but also in response to negative or neutral stimuli (Gruber, 2011). Thus, the everyday positive emotions experienced by patients with bipolar disorder may be more resistant to change (denser positive emotion network) than is the case for healthy control individuals. Investigators might also profitably examine temporal connections between specific emotions. For example, researchers interested in studying MDD and general anxiety disorder (GAD), two highly comorbid disorders (Kessler, Chiu, Demler, \& Walters, 2005), might investigate the temporal relations between anxiety and sadness (e.g., anxiety at time $t-1$ to sadness at time $t$ ) and how these associations may differ between individuals with GAD or MDD. For example, it is possible that both clinical groups would have a stronger absolute temporal relation between anxiety and sadness than would healthy control individuals. Alternatively, MDD and GAD patients may not differ in their absolute temporal relation between anxiety and sadness but may differ in the directionality of this association, with GAD individuals experiencing an increase in sadness after feeling anxious and MDD individuals experiencing a decrease in sadness after feeling anxious.
Our findings are important for understanding the emotional disturbances at play in MDD. Combined with previous empirical work (Kuppens et al., 2010; Rottenberg et al., 2005), they may suggest that in MDD, emotions, and in particular negative emotions, have become decoupled of their adaptive function to flexibly respond to changes or regulation efforts. This inflexibility prevents the individual from adaptively responding emotionally to ongoing events and creates a spiral of mutually reinforcing emotions that, as it seems, start to have a life of their own instead of responding to what happens in the outside world. It is not coincidental that a number of novel and promising approaches to the treatment of MDD (e.g., acceptance and commitment therapy or mindfulnessbased programs; Hayes, Luoma, Bond, Masuda, Lillis, 2006) take this as a starting point in the sense that they focus on helping people to become more broadly attuned to what is happening in the present moment rather than being absorbed in their internal states.

In conclusion, by examining the emotion network in MDD, we illustrated how everyday emotional experiences in MDD mutually reinforce each other across time. The results of this study clearly indicate that relative to healthy control individuals, individuals with MDD are characterized by a denser negative emotion network, thereby reflecting a negative emotion system that is more resistant to change.

\section{Author Contributions}

M. L. Pe, K. Kircanski, P. Kuppens, F. Tuerlinckx, and I. H Gotlib contributed to the study concept. R. J. Thompson, J. Mata, S. M. Jaeggi, M. Buschkuehl, J. Jonides, and I. H. Gotlib conceptualized the experience sampling study and provided the data for the present study. M. L. Pe and L. F. Bringmann analyzed the data, and $M$. Mestdagh rechecked the data analyses. M. L. Pe, P. Kuppens, and I. H. Gotlib interpreted the results. M. L. Pe and K. Kircanski drafted the manuscript, and P. Kuppens, R. J. Thompson, and I. H. Gotlib critically revised the manuscript. All authors approved the final version of the manuscript for submission.

\section{Acknowledgments}

The authors thank Patricia J. Deldin for her help in study design and Courtney Behnke, Sarah Victor, Brooke Gilbert, and Charishma Chotalia for their assistance in project management.

\section{Declaration of Conflicting Interests}

The authors declared that they had no conflicts of interest with respect to their authorship or the publication of this article.

\section{Funding}

This research was supported by National Institute of Mental Health Grants MH60655 (to J. Jonides), MH59259 (to I. H. Gotlib), F32 MH091831 (to R. J. Thompson), and F32 MH096385 (to K. Kircanski); the Deutsche Forschungsgemeinschaft Fellowship 
(Wi3496/4-1 to J. Mata); the Research Council of KU Leuven (GOA/15/003 to F. Tuerlinckx and P. Kuppens); the Interuniversity Attraction Poles programme financed by the Belgian government (IAP/P7/06); and the Fonds Wetenschappelijk Onderzoek-Vlaanderen Travel Grant K206813N (to M. L. Pe).

\section{Notes}

1. The term density is used in the literature on mathematics of networks. It refers to the number of connections between nodes (or vertices) in a given network (Newman, 2010). In the present study, rather than counting the number of connections, we examined the mean strength of the connections between nodes (i.e., emotions).

2. In contrast, research on emotional responses to events in daily life has shown that persons with MDD report a greater emotional change in response to positive events than do nondepressed persons (e.g., Bylsma, Taylor-Clift, \& Rottenberg, 2011; Thompson et al., 2012). It is important to note, however, that events reported in daily life are subjective. When participants report a negative or positive event, they have already interpreted the event as such; the self-report does not give information concerning whether a more objectively rated negative or positive event has occurred. In the emotion-network density framework, we consider reactivity to objective events, which is aligned more closely with how reactivity is measured in the laboratory, typically in response to objective stimuli.

3 . We reran the analyses including the outliers, and the results remained the same.

4. Because emotion variables tend to be highly correlated with each other, and because we included all of the emotion variables at time $t-1$ as predictors, suppressor effects may have influenced our findings. To ensure that this was not the case, we ran 121 simple multilevel models, each of which included only one predictor at a time. Our results remained largely similar, with the exception that analyses of the positive emotion network yielded slightly different results: Specifically, the CTL group $(M=0.10, S E=0.00)$ had a denser positive emotion network than did the MDD group $(M=0.09, S E=0.00), t(100)=$ $-2.57, p=.01, d=0.51$. However, because this method of analysis increases the risk of inflating the slope estimates, thereby leading to inflated density estimates, we caution readers when interpreting results from this analysis.

5. We also examined whether BDI-II scores are related to network density. We computed correlations separately for the MDD and CTL participants because BDI-II scores were used in part to assign participants to diagnostic groups and, therefore, had a bimodal distribution. Because the BDI-II scores for the CTL participants were highly skewed, we log-transformed these scores. Results were similar for both CTL and MDD individuals: BDI-II scores were positively correlated with the density of the overall network (MDD: $r=.38, p<.01$; CTL: $r=.27, p=.05$ ) and the negative emotion network (MDD: $r=.33, p=.02$; CTL: $r=.33, p=.02$ ), whereas this was not the case for the positive emotion network (MDD: $r=.04, p=.76$; CTL: $r=.23, p=.11$ ). 6. We also examined whether recruitment site (i.e., University of Michigan, Stanford University) or age affected these group differences. Age did not moderate these group differences, $F(1$, 100) $=0.95, p=.33, \eta_{p}{ }^{2}=.01$; however, site did moderate these group differences, $F(1,100)=4.34, p=.04, \eta_{p}{ }^{2}=.04$. Within the
MDD group, a larger difference between the negative and positive emotion networks was found in Stanford than in Michigan, $t(51)=-2.47, p=.02, d=-0.69$; this was not the case within the CTL group, $t(49)=0.16, p=.87, d=0.05$. More important, however, separate analyses of both sites consistently yielded significant group differences in the density of the negative emotion network, Michigan: $t(52)=7.37, p<.01, d=2.05$; Stanford: $t(48)=10.65, p<.01, d=3.07$, and no group differences in the density of the positive emotion network-Michigan: $t(52)=$ 0.42, $p=.68, d=0.12$; Stanford: $t(48)=0.92, p=.36, d=$ -0.26 . Thus, results remained consistent even after we examined group differences in the densities of the emotion networks independently for each site.

7. To investigate whether the diagnostic group difference in negative emotion-network density is stronger in women than in men, we ran a regression with negative emotion-network density as the dependent variable and group, gender, and its interaction as predictors. No significant interaction effect was found, which indicated that the difference between diagnostic groups did not differ between genders.

8. The standard-deviation score was computed by calculating the within-person standard deviation of each emotion variable first and then aggregating the mean of these standard deviations across emotions.

9. An alternative way to control for group differences in variance is to standardize all the emotion variables within persons (i.e., within a single person, the mean is 0 and variance is 1) and conduct the same multilevel models discussed earlier on these standardized variables. We conducted these analyses with similar results. The MDD group still had a denser overall emotion network than did the CTL group, $t(104)=4.81, p<.001$, $d=0.94$. Again, this result was driven by a denser negative emotion network in the MDD participants, $t(104)=4.91, p<$ $.001, d=0.96$; the two groups did not differ in density of the positive emotion network, $t(104)=1.26, p=.21, d=0.25$.

10. We clarify that positive and negative emotion networks are defined as networks in which positive and negative emotions are used as dependent variables, respectively, and the predictors for both networks are all of the different emotions at time $t-1$.

11. The strong influence of the cross-lagged relations on the group difference in density findings suggests that the denser negative emotion network found in MDD is more about the insulated and self-determining nature of the negative emotion system and less about the perseveration of negative affect (which is measured by the autocorrelation or inertia variable).

\section{References}

American Psychiatric Association. (2013). Diagnostic and statistical manual of mental disorders (5th ed.). Arlington, VA: American Psychiatric Publishing.

Barrett, D. J., \& Feldman Barrett, L. (2000). ESP: The experience sampling program (Version 4.0) [Software]. Available at http://www.experience-sampling.org/

Beck, A. T., Steer, R. A., \& Brown, G. K. (1996). Beck Depression Inventory-II (2nd ed.). San Antonio, TX: Psychological Corporation.

Borsboom, D. (2008), Psychometric perspectives on diagnostic systems. Journal of Clinical Psychology, 64, 1089-1108. 
Bringmann, L. F., Vissers, N., Wichers, M., Geschwind, N., Kuppens, P., Peeters, F., . . Tuerlinckx, F. (2013). A network approach to psychopathology: New insights into clinical longitudinal data. PLOS ONE, 8(4), e60188. Retrieved from http://www.plosone.org/article/info\%3Adoi\%2F10 $.1371 \% 2$ Fjournal.pone.0060188

Bylsma, L. M., Morris, B. H., \& Rottenberg, J. (2008). A metaanalysis of emotional reactivity in major depressive disorder. Clinical Psychology Review, 28, 676-691.

Bylsma, L. M., Taylor-Clift, A., \& Rottenberg, J. (2011). Emotional reactivity to daily events in major and minor depression. Journal of Abnormal Psychology, 120, 155-167.

Cramer, A. O., Waldorp, L. J., van der Maas, H. L., \& Borsboom, D. (2010). Comorbidity: A network perspective. Behavioral E Brain Sciences, 33, 137-150.

First, M. B., Spitzer, R. L., Gibbon, M., \& Williams, J. B. W. (2001). The Structured Clinical Interview for DSM-IV-TR Axis I Disorders. New York: Biometrics Research, New York State Psychiatric Institute.

Gruber, J. (2011). Can feeling too good be bad? Positive emotion persistence (PEP) in bipolar disorder. Current Directions in Psychological Science, 20, 217-221.

Hayes, S. C., Luoma, J. B., Bond, F. W., Masuda, A., \& Lillis, J. (2006). Acceptance and commitment therapy: Model, processes and outcomes. Behaviour Research and Therapy, $44,1-25$.

Houben, M., Van den Noortgate, W., \& Kuppens, P. (2013). The relation between patterns of emotional change and psychological well-being: A meta-analysis. Manuscript submitted for publication.

Izard, C. E. (2009). Emotion theory and research: Highlights, unanswered questions and emerging issues. Annual Review of Psychology, 60, 1-25.

Kashdan, T. B., \& Rottenberg, J. (2010). Psychological flexibility as a fundamental aspect of health. Clinical Psychology Review, 30, 865-878.

Kendler, K. S., Zachar, P., \& Craver, C. (2011). What kinds of things are psychiatric disorders? Psychological Medicine, 41, 1143-1150.
Kessler, R. C., Chiu, W. T., Demler, O., \& Walters, E. E. (2005). Prevalence, severity, and comorbidity of 12-month DSM-IV disorders in the National Comorbidity Survey Replication. Archives of General Psychiatry, 62, 617-627.

Koval, P., Kuppens, P., Allen, N. B., \& Sheeber, L. B. (2012). Getting stuck in depression: The roles of rumination and emotional inertia. Cognition E Emotion, 26, 1412-1427.

Kuppens, P., Allen, N. B., \& Sheeber, L. B. (2010). Emotional inertia and psychological maladjustment. Psychological Science, 21, 984-991.

Kuppens, P., Sheeber, L. B., Yap, M. B., Whittle, S., Simmons, J. G., \& Allen, N. B. (2012). Emotional inertia prospectively predicts the onset of depressive disorder in adolescence. Emotion, 12, 283-289.

Larsen, R., \& Diener, E. (1987). Affect intensity as an individual difference characteristic: A review. Journal of Research in Personality, 21, 1-39.

Newman, M. E. J. (2010). Networks: An introduction. New York, NY: Oxford University Press.

Nolen-Hoeksema, S. (2001). Gender differences in depression. Current Directions in Psychological Science, 10, 173-176.

Nolen-Hoeksema, S., Wisco, B. E., \& Lyubomirsky, S. (2008). Rethinking rumination. Perspectives on Psychological Science, 3, 400-421.

Pe, M. L., \& Kuppens, P. (2012). The dynamic interplay between emotions in daily life: Augmentation, blunting, and the role of appraisal overlap. Emotion, 12, 1320-1328.

Rottenberg, J. (2005). Mood and emotion in major depression. Current Directions in Psychological Science, 14, 167-170.

Rottenberg, J., Gross, J. J., \& Gotlib, I. H. (2005). Emotion context insensitivity in major depressive disorder. Journal of Abnormal Psychology, 114, 627-639.

Russell, J. A. (2003). Core affect and the psychological construction of emotion. Psychological Review, 110, 145-172.

Thompson, R. J., Mata, J., Jaeggi, S. M., Buschkuehl, M., Jonides, J., \& Gotlib, I. H. (2012). The everyday emotional experience of adults with major depressive disorder: Examining emotional instability, inertia, and reactivity. Journal of Abnormal Psychology, 121, 819-829. 